\title{
Hen The application of XRT in the De Beers Group of companies
}

\author{
by A. Voigt*, G. Morrison*, G. Hill*, G. Dellas*, and \\ R. Mangera*
}

\section{Synopsis}

X-ray transmission (XRT) sorting has become the preferred recovery technology option in several parts of the diamond-winning flow sheet. In the De Beers Group, applications of XRT are found across kimberlite, alluvial, and marine operations. This is the result of intensive $R \& D$ conducted over the years to arrive at a suite of machine embodiments capable of sorting and auditing diamonds across all size ranges.

The first applications in the marine environment used the technology in an auditing mode, and served as a useful early predictor of diamond content weeks ahead of sorthouse returns. The same machines are now available with ejection capability to produce high final product grades. The next application was tests on coarse alluvial gravels as an alternative to dense medium separation. The results were very encouraging, and tests are planned for both green- and brownfield kimberlite environments, as well as to explore an alternative to conventional techniques in final diamond recovery. At the Jwaneng mine Large Diamond Pilot Plant (LDPP), the objective is to recover diamonds in the size fraction -45 $+25 \mathrm{~mm}$.

The technical challenge remains in the finer sizes, for high-capacitymachines as direct alternatives to conventional diamond recovery technologies. This is an area of ongoing $R \& D$ and it is only a matter of time before the breakthrough emerges.

\section{Keywords}

XRT, diamonds, De Beers, DMS, sorting.

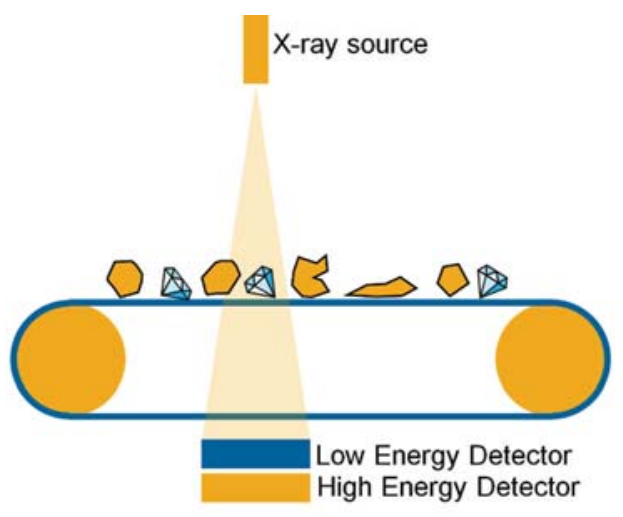

Figure 1-Schematic of a dual-energy line-scan system
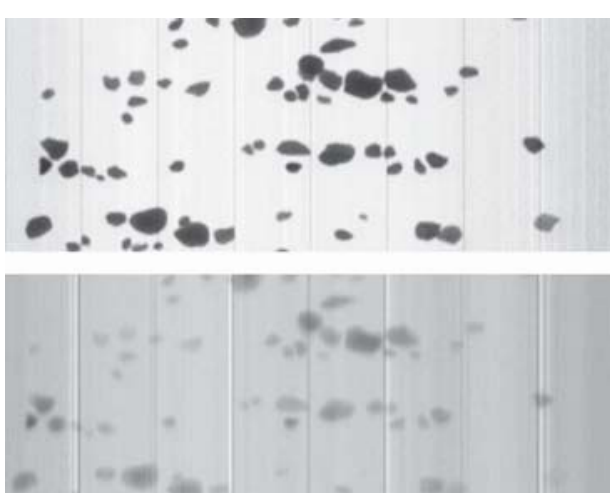

Figure 2-Examples of low-energy (top) and highenergy (bottom) images of diamondiferous material passing the $\mathrm{X}$-ray line-scan camera

two sensors, one responding to low-energy Xrays and one to high-energy $\mathrm{X}$-rays. Feed material can be imaged either while on the belt, or while in flight.

Examples of raw X-ray images of diamondiferous feed material are given in Figure 2. Particles appear as shadows in the Bergmann, 2010; Sasman, Deetlefs, and van der Westhuyzen, 2018). Dual-energy XRT (DE-XRT), in which images of the target material are obtained at both high and low Xray energies, allows for elemental analysis and therefore can be used to discriminate between various minerals.

A DE-XRT system makes use of a dualenergy X-ray line scan sensor to generate images of transmitted X-rays (Figure 1). Dualenergy refers to the camera, which contains
* De Beers Technologies (SA), Johannesburg, South Africa.

(c) The Southern African Institute of Mining and Metallurgy, 2019. ISSN 2225-6253. This paper was first presented at the Diamonds - Source to Use 2018 Conference, 11-13 June 2018, Birchwood Hotel and OR Tambo Conference Centre, JetPark, Johannesburg, South Africa. 


\section{The application of XRT in the De Beers Group of companies}

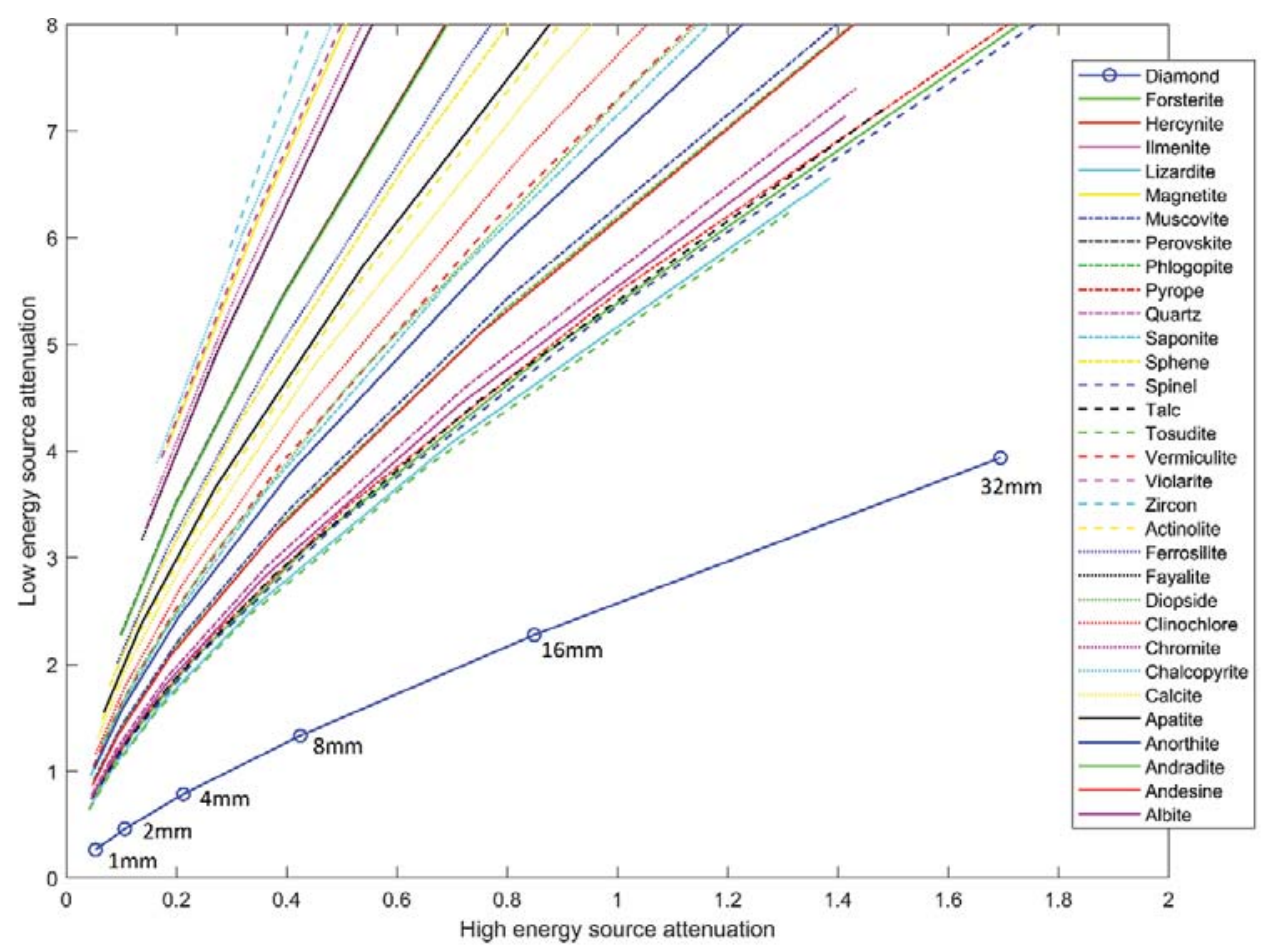

Figure 3-Result of a theoretical study of how various minerals absorb high- and low-energy X-rays. Diamonds can be distinguished from other minerals based on their absorption of high- and low-energy $\mathrm{X}$-rays

image, as X-rays are absorbed by the particles and fewer Xrays arrive at the detector. The shadows appear darker in the low-energy image than the high energy-image, since lowenergy X-rays are more readily absorbed than high-energy $\mathrm{X}$-rays.

The extent to which materials absorb X-rays depends on the elements present, density and thickness of the material, as well as the characteristics of the X-rays. Each mineral absorbs high- and low-energy X-rays in different ratios. Figure 3 shows the theoretically predicted absorption of highand low-energy X-rays for various minerals of varying thickness, derived from the material properties (XCOM, 2018). It is evident that diamond can be distinguished from other minerals. Note that Figure 3 represents the ideal case. In practice, detector noise and other variations result in imperfect discrimination. This is especially true for small particles.

The high- and low-energy images can be combined, and each pixel coloured according to its classification as shown in Figure 4.

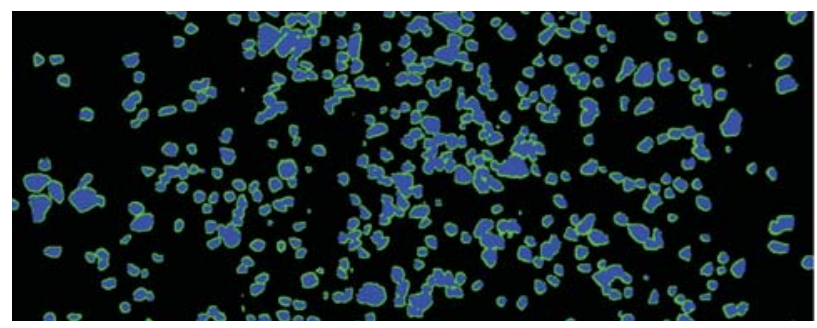

Figure 4- In this classified image, green regions represent diamond and blue non-diamond. The green rims around non-diamond particles are regions where the material is too thin to be classified correctly
Image processing techniques are used to identify particles in the classified image. Various tests are carried out on each particle before it is identified as a target or non-target. In a sorting machine embodiment, the target particles are typically ejected from the material stream using an air ejection system.

In addition to identifying particles, the XRT absorption images contain information from which the size and mass of particles can be inferred. This allows for diamond auditing applications, which are discussed further below.

\section{A summary of XRT in the De Beers Group}

The first XRT system in De Beers was developed by the De Beers Diamond Trading Company in 1990. This is a laboratory system used for detecting and analysing liberated diamonds in the Kimberley Microdiamond Laboratories, and a version is still in use today.

Following this, in the early 1990s, the De Beers Mineral Processing Division in Johannesburg evaluated XRT as one of the technologies for detecting unliberated diamonds within kimberlite. At that time, fast neutron radiography was identified as the preferred method for detecting these unliberated stones. During this work, XRT again showed great potential for the detection of liberated diamonds, although the commercially available excitation and detection technology was not yet mature enough for application to kimberlite processing or diamond recovery.

The major advantages that XRT offered were:

$>$ All diamond types would be recovered with no concerns regarding low-luminescing stones

> Ultra-low yields would be possible, making new flow sheet designs feasible

$>$ Lower sensitivity to dust and masking effects than conventional optical techniques. 


\section{The application of XRT in the De Beers Group of companies}

However, challenges remained for XRT on multiple fronts (as described later by Buxton and Crosby, 2008):

> Predicted throughputs were low at the targeted size ranges

> The robustness of the enabling technology was poor

> The cost of developing a viable sorter to compete with existing equipment was prohibitive

> Reliable performance benchmarks were nonexistent, and thus trust was low.

Nevertheless, during the next decade the underlying excitation, detection, and data processing technologies made steady progress and the development of viable sorting machines became a possibility in the mid-2000s.

The following application areas for XRT were identified and pursued:

> To provide online diamond auditing information. The first XRT auditing trials were run in 2013 on offshore marine vessels.

> To complement X-ray luminescence (XRL) sorting machines in the recovery plants to produce concentrates containing a high weight percentage of diamonds. XRT reconcentration machines have been operating on offshore marine vessels since 2016.

> As a low-OPEX alternative to coarse DMS that would be insensitive to feed density. The first XRT pilot plant was operational in 2014 at Namdeb.

> To recover large diamonds early in the flow sheet. The Jwaneng mine LDPP will operate from 2018 to 2019.

Going forward, challenges remain as regards fine diamond recovery, particularly with wet fine material. In addition, applications in the re-deployable and ultra-coarse domains are being watched with interest and may be able to unlock further value for De Beers.

\section{Marine auditing and sorting applications}

XRT can provide real-time estimates of diamond size frequency distributions (DSFD). This is because high-

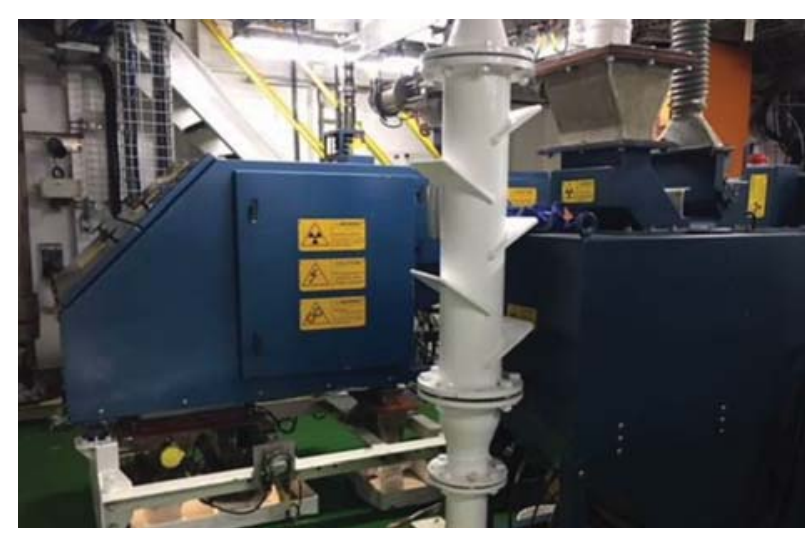

Figure 5-XRT reconcentration machine (BDT1122) installed on a Debmarine Namibia mining vessel. The machine processes the final recovery plant concentrate before canning

resolution images of the feed material are captured and the absolute X-ray absorption of a particle depends on its size and mineralogy. The carat mass of individual diamonds can therefore be estimated as they are processed.

Real-time DSFD information provides particular benefit in the marine mining environment. In a typical marine operation, the sea floor is systematically mined in welldefined sections. The final concentrates, usually the product of X-ray reconcentration unit processes, are canned on board. Consignments are exported to land on a regular basis, where they are sorted and sized in a hand-sorting facility. It is only at the end of this process (which can take several days) that the recovery statistics for a particular mining area become known. Plant metallurgists rely on proxy measures, such as the number of ejections recorded by the diamond recovery machines. These measures vary significantly depending on the machine set-up parameters and the mineralogy of gangue material being fed to the circuit.

De Beers Marine (DBM), De Beers Marine Namibia (DBMN), and De Beers Technologies (SA) began a collaborative XRT test programme in 2013. A mine test unit

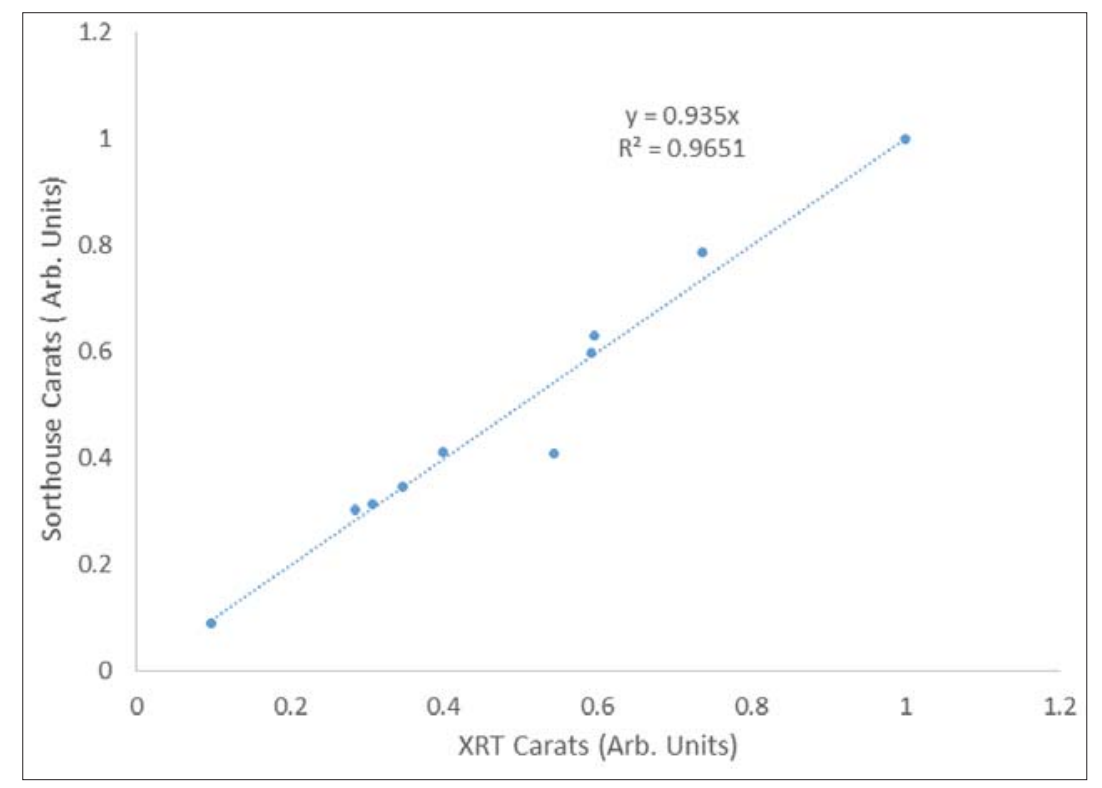

Figure 6-Correlation between the total carats recovered by the sorthouse and the XRT estimate 


\section{The application of XRT in the De Beers Group of companies}

(MTU) was installed on the Debmarine Namibia DebMar Atlantic Mining Vessel. The final concentrate underwent XRT analysis before being canned for export.

Over a one-year test period, the XRT estimate of DSFD and total carat content was compared with the sorthouse results. The tests were successful, showing that XRT provides an accurate and timeous estimate of the DSFD and carat content of the final concentrate. The XRT unit process (BDT1122) has since been commercialized and installed on five DBMN mining vessels as shown in Figure 5. The machines are available in either an auditing configuration (providing carat and DSFD information), or a reconcentration configuration (where sorting functionality is included).

Figure 6 shows a typical correlation between carats recovered in the sorthouse versus the total carat content estimated by XRT, for +3 ds material. XRT generally provides an accurate estimate of total carats recovered, and has replaced other proxy methods as a real-time measure of recoveries on many marine operations.

While the application of XRT in the marine mining environment has proved useful so far, a few challenges remain. These are primarily associated with the detection of fine ( $-3 \mathrm{ds}$ or $-1.3 \mathrm{~mm}$ square mesh sieve) material. Such material is at the limit of the resolution capability and noise characteristics of dual-energy X-ray detectors. In order to ensure that fine diamonds are detected, and recovered, the algorithm parameters are such that a significant number of false detections occur, increasing the yield on the finer material. Indeed, the outlier cases seen in Figure 6 can be associated with a period that had a high incidence of dusty or gritty material in the feed. In such cases, XRT overestimates the carat content.

The reconcentration version of the machine (BDT1122) includes a multi-channel ejection system. Its purpose is to provide a high diamond by weight (DBW) product, and it is being piloted at various marine and land-based plants. The same challenge exists with fine material, as discussed in the previous paragraph.

Various initiatives are underway to address the challenges presented above. These include improved dust and grit removal systems, as well as higher resolution X-ray camera technology.

\section{XRT as an alternative to dense medium separation}

High-throughput XRT machines were identified as a lower

\section{Table I \\ An illustrative comparison of operating costs when replacing a coarse DMS with an XRT solution treating the same throughput. Values are normalized to the total DMS cost}

\begin{tabular}{|l|c|c|}
\hline \multirow{2}{*}{ Cost item } & \multicolumn{2}{|c|}{ Normalized cost per ton } \\
\cline { 2 - 3 } & XRT & DMS \\
\hline Electricity & $14 \%$ & $36 \%$ \\
Labour & $11 \%$ & $8 \%$ \\
Maintenance and consumables & $7 \%$ & $32 \%$ \\
Downstream costs & $2 \%$ & $23 \%$ \\
\hline Total & $34 \%$ & $100 \%$ \\
\hline
\end{tabular}

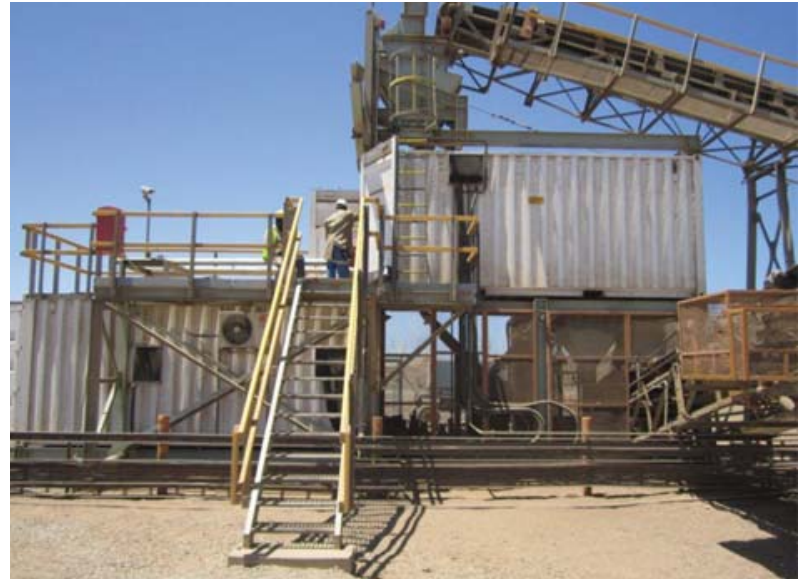

Figure 7-The XRT pilot plant installation in Oranjemund, Namibia. The system consisted of a containerized high-throughput machine coupled with a container housing the services required to run the plant

cost alternative to DMS. These machines are predicted to run at lower operating costs and to produce lower and more consistent yields that will result in downstream treatment cost benefits and simplifications. A comparison is shown in Table I.

During 2014 to 2016, Namdeb and De Beers Technologies SA collaborated to run a joint pilot test programme with the goal of evaluating high-throughput XRT in the alluvial application as an alternative to DMS plants.

Figure 7 shows the installation in Oranjemund. A number of novel operational features were evaluated, including feed rate control loops, and generation of DSFD information.

The machine used in the tests was the De Beers Technologies SA XRT Coarse Concentrator (XRT-CC) BWT1186. This containerized sorting machine has a $900 \mathrm{~mm}$ wide belt and is designed to process material from $6 \mathrm{~mm}$ to $75 \mathrm{~mm}$.

The programme tested the technology with material in size ranges between $6 \mathrm{~mm}$ and $32 \mathrm{~mm}$. Dry and wet materials were specifically tested separately to determine the effect of moisture on sorting performance. In addition, as this was an alluvial operation, the impact of marine shell in the feed was also investigated. The test programme set out to determine the following critical parameters:

> The optimal feed rate per size fraction

- Diamond recovery efficiency at these throughputs

- The availability and reliability of the XRT technology

- The operating costs of the XRT technology.

The results indicated that the XRT-CC machine is well suited as an alternative to coarse DMS. The tests also showed that feed preparation is important as increased undersize material in the feed reduces the throughput to less than the optimum for $100 \%$ diamond recovery. Wet fine material required special consideration due to the material sticking to the belt, leading to either higher-than-expected yield (for topdown ejection systems) or more frequent maintenance (for bottom-up ejection systems). It is worth noting that these findings are not XRT-specific concerns but would apply to any belt-fed sensor sorting system. 


\section{The application of XRT in the De Beers Group of companies}

\section{Large diamond recovery}

The high throughput and low yielding performance of XRT sorting machines enable them to be applied upstream of existing concentration circuits to target the large liberated diamonds above the conventional top particle cut-off size. This configuration allows this revenue to be recovered as early as possible and reduces the probability of damage to the large stones (Sasman, Deetlefs, and van der Westhuyzen, 2018).

The LDPP at Jwaneng mine in Botswana (Figure 8) will undergo a 12-month test programme during 2018 and 2019 to quantify the occurrence rate of large diamonds at that operation. The plant will treat the $25 \mathrm{~mm}$ to $45 \mathrm{~mm}$ scrubber oversize size fraction with two De Beers Technologies (SA) BWT11162 machines. This containerized sorting machine has an $1800 \mathrm{~mm}$ wide belt and can process material from 6 $\mathrm{mm}$ to $75 \mathrm{~mm}$.

\section{Alternatives for coarse diamond recovery post-DMS}

As much as the application of high-capacity XRT machines is seen as an alternative to DMS and upfront large diamond recovery, it also enables alternative flow sheet configurations (Valbom and Dellas 2010). Venetia mine is installing a De Beers Technologies (SA) XRT-CC machine after DMS, targeting the coarse $(-25+8 \mathrm{~mm})$ size fraction, as shown in Figure 9.

As feed to the plant becomes harder and denser, and with varying PSDs, the limitations of the current flow sheet configuration have become evident. High-throughput XRT technology will alleviate both the capacity and PSD variation challenges by a simple modification of the flow sheet as shown in Figure 9.

The main advantage of the new configuration is the separation of the coarse circuit from the final recovery plant into a standalone high-capacity circuit. The recovery plant will then be reconfigured for the fines and middles size fractions at improved capacity.

\section{Fine diamond recovery at high throughput}

XRT machines that treat fine material could find application in the recovery plant and as a replacement for fines DMS.

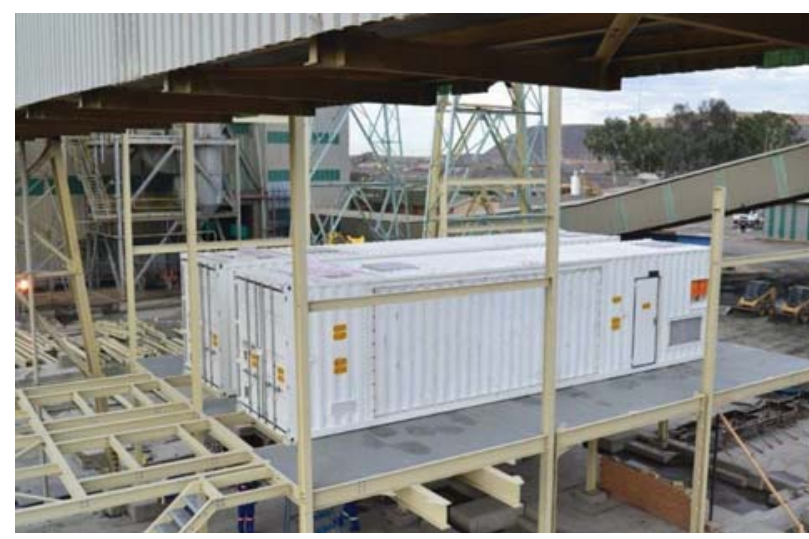

Figure 8-The Large Diamond Pilot Plant installation at Jwaneng mine, Botswana, showing the two XRT machines side-by-side. Containerized machines simplify shipping and installation and enable pre-

commissioning to be done at the factory, which shortens the on-site commissioning time
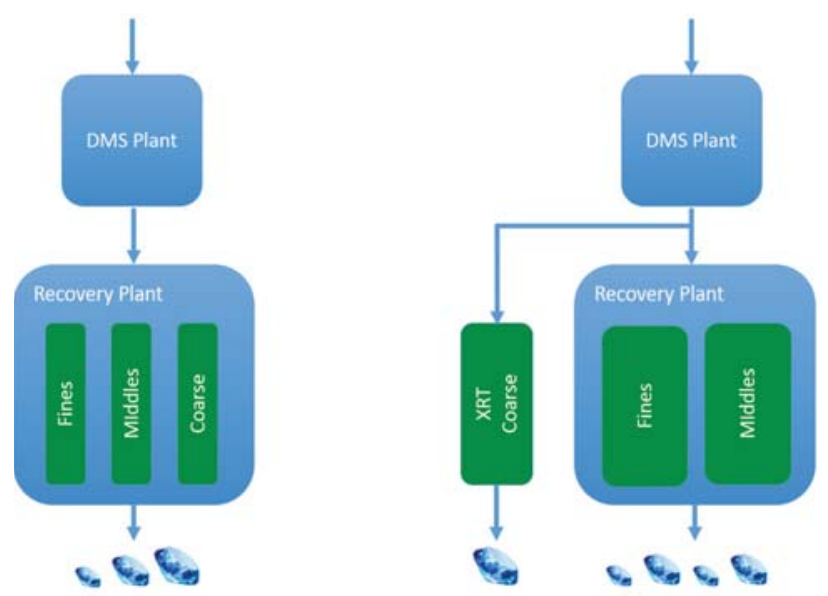

Figure 9-The current (left) and modified (right) flow sheets at Venetia mine, South Africa. Currently, the recovery plant treats all size fractions - fines, middles, and coarse - on dedicated circuits. The modified flow sheet includes XRT technology that treats the coarse fraction independently from the recovery plant, which is now reconfigured for the fines and middles fractions only

However, the throughput requirements in the DMS application make any sensor-based sorting system (including XRT) commercially unviable due to the current CAPEX and OPEX estimations for such projects.

Apart from the commercial considerations, the treatment of fine material at high throughput presents various technical challenges to sensor-based sorting machines. While the demand placed on the detection and classification system is highest, the feed presentation system and the ejection system are also strained.

\section{Feed presentation}

Systems dealing with large particles are fairly robust to dust and grit that is entrained with the material. However, as the size fraction being treated becomes smaller the detection system is less able to operate effectively. Added to this is the challenge of feeding fine, wet material which sticks to the belt and tends to clump. This adversely affects the recovery efficiency and yield.

\section{Detection and classification}

The biggest challenge presented by finer material relates to requirements placed on the detection system. Difficulties arise because finer particle sizes require smaller pixels on the Xray sensor. To ensure that a reasonable signal is achieved on these smaller pixels, a number of engineering solutions are possible.

> Increasing the signal integration time, resulting in reduced throughput due to the associated reduction in the speed of the material passing the sensor

> Bringing the X-ray source closer to the sensor, resulting in reduced throughput due to reduced belt width

> Increasing the X-ray power, placing greater pressure on the engineering of X-ray safety and resulting in increased failure rates of the X-ray generation and detection components. 


\section{The application of XRT in the De Beers Group of companies}

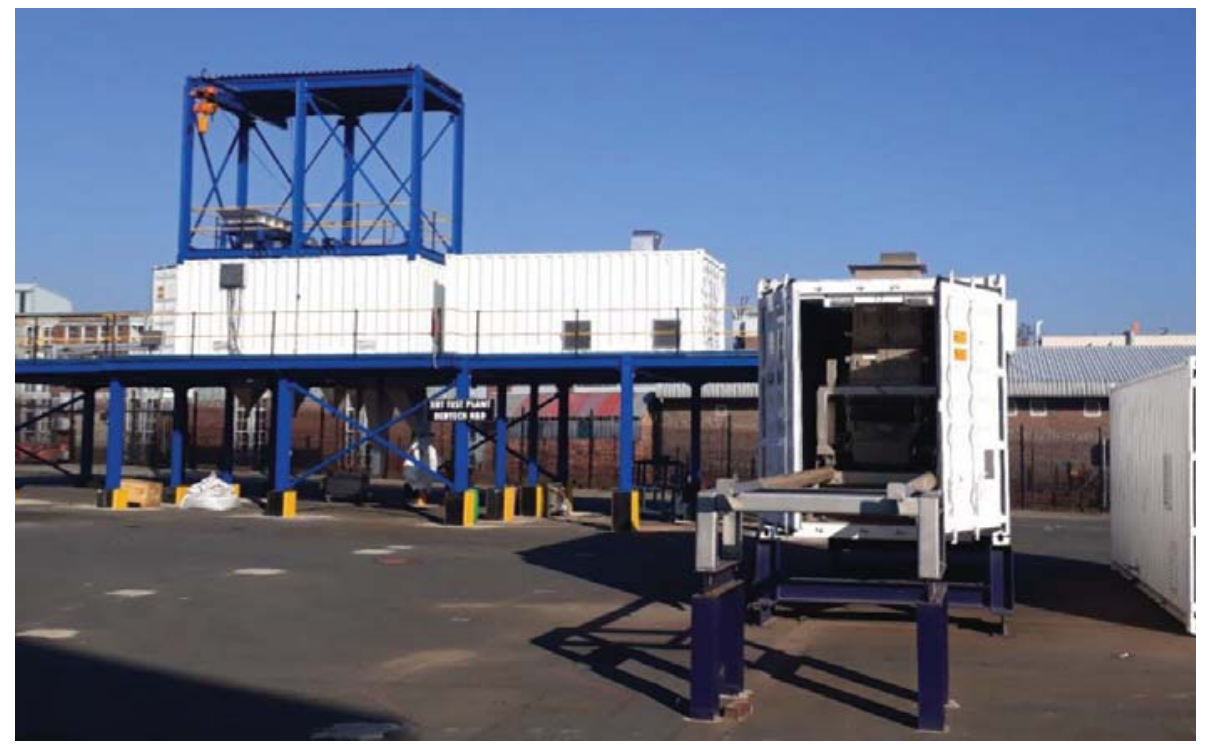

Figure 10-The XRT test plant at the De Beers Technologies (SA) campus, Johannesburg

To achieve high throughput when treating fine material, a much higher data processing rate is required. This is due to the finer detector resolution, which requires a high number of small pixels. Another challenge associated with small pixels is that the signal-to-noise level is decreased. The resulting data is noisier, which makes the task of the classification system more difficult.

\section{Ejection}

For handling fine material, the ejection system needs to be more precise. Ejectors can be thought of as having a footprint - the bigger this footprint, the greater the number of 'passenger' particles that will be recovered with each ejection of a target particle. As the material becomes finer, the ejection footprint needs to be reduced, otherwise the grade of the recovered material will be too low.

\section{Test facility in Johannesburg}

A permanent and secure XRT test facility is located at the De Beers Technologies (SA) campus in Johannesburg (Figure 10) and forms part of the Mineral Sample Treatment Plant (MSTP). Several performance parameters, including recovery efficiency, yield, throughput, and size range, as well as the interaction between these parameters, can be studied under controlled conditions.

\section{Conclusions}

XRT technology has had a positive, expanding impact across the De Beers Group. As we have discussed in this paper, this covers a wide range of applications from auditing in the marine environment to large diamond recovery at Jwaneng mine.

The remaining challenges are associated with the fine diamond processing applications, particularly with wet fine material. Potential applications in the re-deployable and ultra-coarse domains are being considered, and may be able to unlock further value for De Beers.

\section{Acknowledgements}

Sincere gratitude goes to the following: De Beers Consolidated Mines Venetia mine, Debmarine Namibia, Debmarine South Africa, Debswana Corporate Centre, Debswana Jwaneng mine, and Namdeb Diamond Corporation.

\section{References}

Buxton, M. and Crosby, R. 2008. Ore sorting - An Anglo American perspective. Proceedings of Sensor Based Sorting 2008, Aachen, Germany. GDMB, Clausthal-Zellerfeld, Germany.

Martz, H.E., Logan, C.M., Schneberk, D.J., and Shull, P.J. 2016. X-Ray Imaging: Fundamentals, Industrial Techniques and Applications. CRC Press.

OWADA, S. 2014. SBS technology for creating environment friendly process of various metals recycling - Advances and problems to be solved. Proceedings of Sensor Based Sorting 2014, Aachen, Germany. GDMB Clausthal-Zellerfeld, Germany. pp. 11-23.

Sasman, F., Deetlefs, B., and van der Westhuyzen, P. (2018). Application of diamond size frequency distribution and XRT technology at a large diamond producer. Journal of the Southern African Institute of Mining and Metallurgy, vol. 118, no. 1. pp. 1-6.

Valbom, D.M.C. and Dellas, G. 2010. State of the art recovery plant design. Proceedings of Diamonds - Source to Use 2010. Southern African Institute of Mining and Metallurgy, Johannesburg. pp. 243-252.

Von Ketelhodt, L. and Bergmann, C. 2010. Dual energy X-ray transmission sorting of coal. Journal of the Southern African Institute of Mining and Metallurgy, vol. 110, no. 7. pp. 371-378.

XCOM. 2018. National Institute of Standards and Technology Physical Measurement Laboratory X-ray cross-sections. https://physics.nist.gov/PhysRefData/Xcom/html/xcom1.htm [accessed 3 March 2018]. 